Annales Academiæ Scientiarum Fennicæ

Mathematica

Volumen 37, 2012, 161-174

\title{
HILBERT MATRIX OPERATOR ON SPACES OF ANALYTIC FUNCTIONS
}

\author{
Bartosz Łanucha, Maria Nowak and Miroslav Pavlović \\ Uniwersytet Marii Curie-Skłodowskiej w Lublinie, Instytut Matematyki \\ pl. M. Curie-Skłodowskiej 1, 20-031 Lublin, Poland; bartosz.lanucha@gmail.com \\ Uniwersytet Marii Curie-Skłodowskiej w Lublinie, Instytut Matematyki \\ pl. M. Curie-Skłodowskiej 1, 20-031 Lublin, Poland; mt.nowak@poczta.umcs.lublin.pl \\ Univerzitet u Beogradu, Matematički Fakultet \\ Studentski trg 16, 11001 Beograd, p.p. 550, Serbia; pavlovic@matf.bg.ac.rs
}

\begin{abstract}
We consider the action of the Hilbert matrix operator, $H$, on the Hardy space $H^{1}$, weighted Hardy spaces $H_{\alpha}^{p}(\alpha \geq 0)$, Bergman spaces with logarithmic weights, etc. In particular, we extend Diamantopoulos-Siskakis result by proving that $H$ maps $H_{\alpha}^{p}$ into $H_{\alpha}^{p}$ if and only if $\alpha+1 / p<1$. A criterion for $H f$ to belong to $H^{1}$ is given provided the coefficients of $f$ are nonnegative. Also, $H$ maps the $A^{2}$-space with weight $\log ^{\alpha}\left(2 /\left(1-|z|^{2}\right)\right)$ into the ordinary Bergman space $A^{2}$ if $\alpha>3$. Similarly, the Bloch space with logarithmic weight is mapped by $H$ into the ordinary Bloch space.
\end{abstract}

\section{Introduction}

The Hilbert matrix is an infinite matrix $H$ whose entries are $a_{n, k}=(n+k+1)^{-1}$. This matrix induces a linear operator on sequences:

$$
H:\left(a_{k}\right)_{k \in \mathbf{N}_{0}} \longmapsto\left(\sum_{k=0}^{\infty} \frac{a_{k}}{n+k+1}\right)_{n \in \mathbf{N}_{0}} .
$$

The following Hilbert's inequality implies that this operator is well defined and bounded on the space $l^{p}$ of all $p$-summable sequences $(p>1)$.

Theorem 1.1. (Hilbert's inequality [5, Chapter IX]) Suppose $1<p<\infty$. If $\left(a_{k}\right)_{k \in \mathbf{N}_{0}} \in l^{p}$, then

$$
\left(\sum_{n=0}^{\infty}\left|\sum_{k=0}^{\infty} \frac{a_{k}}{n+k+1}\right|^{p}\right)^{\frac{1}{p}} \leq \frac{\pi}{\sin \frac{\pi}{p}}\left(\sum_{k=0}^{\infty}\left|a_{k}\right|^{p}\right)^{\frac{1}{p}} .
$$

Moreover, the constant $\frac{\pi}{\sin \frac{\pi}{p}}$ is best possible.

Apart from sequence spaces, the Hilbert matrix can be viewed as an operator on spaces of analytic functions by its action on their Taylor coefficients. If

$$
f(z)=\sum_{k=0}^{\infty} \hat{f}(k) z^{k}
$$

doi:10.5186/aasfm.2012.3715

2010 Mathematics Subject Classification: Primary 47B37, 30H10, 30H20, 30H30.

Key words: Hilbert matrix, Hardy spaces, Bergman spaces, Bloch and Besov spaces.

The third author is supported by NTR Serbia, Project ON174017. 
is a holomorphic function in the unit disk $\mathbf{D}=\{z \in \mathbf{C}:|z|<1\}$, then we define a transformation $H$ by

$$
H f(z)=\sum_{n=0}^{\infty} \sum_{k=0}^{\infty} \frac{\hat{f}(k)}{n+k+1} z^{n} .
$$

Let $H(\mathbf{D})$ be the algebra of holomorphic functions in D. For $0<p \leq \infty$ Hardy space $H^{p}$ is the space of all holomorphic functions $f \in H(\mathbf{D})$ for which

$$
\|f\|_{p}=\sup _{0 \leq r<1} M_{p}(r, f)<\infty,
$$

where

$$
\begin{aligned}
M_{p}(r, f) & =\left(\frac{1}{2 \pi} \int_{0}^{2 \pi}\left|f\left(r e^{i \theta}\right)\right|^{p} d \theta\right)^{\frac{1}{p}}, \quad 0<p<\infty ; \\
M_{\infty}(r, f) & =\sup _{0 \leq \theta \leq 2 \pi}\left|f\left(r e^{i \theta}\right)\right| .
\end{aligned}
$$

It follows from the Hardy's inequality ([4], p. 48)

$$
\sum_{k=0}^{\infty} \frac{|\hat{f}(k)|}{k+1} \leq \pi\|f\|_{1}
$$

that $H$ is well defined for each $f \in H^{p}, p \geq 1$. It was proved by Diamantopoulos and Siskakis ([1]) that the operator $H$ is bounded on $H^{p}, 1<p<\infty$, and not bounded on $H^{1}$ and $H^{\infty}$. In [3] the following formula for $H$ acting on $H^{p}, p \geq 1$, was noticed

$$
H f=P_{+}\left(M_{b} C f\right),
$$

where $C f\left(e^{i t}\right)=f\left(e^{-i t}\right)$ is an isometry from $H^{p}$ into $L^{p}(\mathbf{T}), M_{b}(u)=b u, b(t)=$ $i e^{-i t}(\pi-t), 0 \leq t<2 \pi$ and $P_{+}$is the Szegö projection given by

$$
P_{+} u(z)=\frac{1}{2 \pi} \int_{0}^{2 \pi} \frac{u(t)}{\left(1-z e^{-i t}\right)} d t, \quad z \in \mathbf{D} .
$$

Recall that the space BMOA consists of the functions $f \in H^{1}$ whose boundary values $f\left(e^{i t}\right)$ are of bounded mean oscillation on $\mathbf{T}$, that is

$$
\sup _{I} \int_{I}\left|f\left(e^{i t}\right)-I(f)\right| d t<\infty
$$

where supremum is taken over all intervals $I \subset \mathbf{T}$ and

$$
I(f)=\frac{1}{|I|} \int_{I} f\left(e^{i t}\right) d t .
$$

If

$$
\lim _{|I| \rightarrow 0} \int_{I}\left|f\left(e^{i t}\right)-I(f)\right| d t=0
$$

then we say that $f \in V M O A$.

Since the space BMOA is the Szegö projection of $L^{\infty}(\mathbf{T})$, we have also the following

Theorem 1.2. The Hilbert matrix operator $H$ acts as a bounded operator from $H^{\infty}$ into BMOA.

The next theorem describes the polynomials that are mapped by $H$ into $V M O A$. 
Theorem 1.3. Let $w$ be a polynomial of degree at least 1. Then $H w \in V M O A$ if and only if $w(1)=0$.

Proof. We know that the operator $H w=P_{+}\left(w\left(e^{-i \theta}\right) b(\theta)\right)$, where $b(\theta)=i e^{-i \theta}(\pi-$ $\theta$ ) for $0 \leq \theta<2 \pi$. The function $b$ is continuous on the unit circle $\mathbf{T}$ except for 1 . If $w(1)=0$, then the function $w\left(e^{-i \theta}\right) b(\theta)$ can be continuously extended on the whole unit circle and $H w$ is the Szegö projection of this continuous function which means that $H w \in V M O A$. It is also clear that if the function $w\left(e^{-i \theta}\right) b(\theta)$ is continuous on $\mathbf{T}$ then $w(1)=0$.

In the next section we show that if $f \in H^{1}$, then $H f$ extends to a continuous function on $\overline{\mathbf{D}} \backslash\{1\}$ and give a sufficient condition for $H f \in H^{1}$. In the case of positive Taylor coefficients we obtain a sufficient and necessary condition for $H f \in$ $H^{1}$. Section 3 is devoted to the weighted Hardy spaces $H_{\alpha}^{p}, 0<p \leq \infty, \alpha>0$, consisting of those $f \in H(\mathbf{D})$ for which $M_{p}(r, f)=O\left((1-r)^{-\alpha}\right)$. We prove that the Hilbert matrix operator is bounded on $H_{\alpha}^{p}$ if and only if $\alpha+1 / p<1$. It is known that the operator $H$ cannot be defined on the Bergman space $A^{2}$ of analytic functions that are square integrable over the unit disk with respect to the Lebesgue area measure. Here we find the subspace of $A^{2}$ which is mapped by $H$ boundedly into $A^{2}$. Finally, we study the acting of the operator $H$ on the Bloch space and Besov spaces.

Throughout the paper the notion $A \asymp B$ means that there exists a positive constant $C$ such that $B / C \leq A \leq C B$.

\section{Hilbert matrix operator acting on $H^{\mathbf{1}}$}

This section contains results on the Hilbert matrix operator acting on $H^{1}$ that are analogous to that obtained for the Libera operator in [12]. The proofs presented here are slightly different from the proofs given in [12].

We start with the following

Lemma 2.1. If $f \in H^{1}$, then $H f$ extends to a continuous function on $\overline{\mathbf{D}} \backslash\{1\}$.

Proof. By (1.2),

$$
H f(z)=\frac{1}{1-z} F_{f}(z)
$$

where

$$
F_{f}(z)=(1-z) \sum_{n=0}^{\infty} \sum_{k=0}^{\infty} \frac{\hat{f}(k)}{n+k+1} z^{n} .
$$

We will show that the function $F_{f}$ can be continuously extended to $\overline{\mathbf{D}}$. For $z \in \mathbf{D}$ we have

$$
\begin{aligned}
F_{f}(z) & =\sum_{n=0}^{\infty} \sum_{k=0}^{\infty} \frac{\hat{f}(k)}{n+k+1} z^{n}-\sum_{n=0}^{\infty} \sum_{k=0}^{\infty} \frac{\hat{f}(k)}{n+k+1} z^{n+1} \\
& =\sum_{k=0}^{\infty} \frac{\hat{f}(k)}{k+1}+\sum_{n=1}^{\infty} \sum_{k=0}^{\infty} \frac{\hat{f}(k)}{n+k+1} z^{n}-\sum_{n=1}^{\infty} \sum_{k=0}^{\infty} \frac{\hat{f}(k)}{n+k} z^{n} \\
& =\sum_{k=0}^{\infty} \frac{\hat{f}(k)}{k+1}-\sum_{n=1}^{\infty} \sum_{k=0}^{\infty} \frac{\hat{f}(k)}{(n+k)(n+k+1)} z^{n}
\end{aligned}
$$


To see that the last double series converges absolutely and uniformly on $\overline{\mathbf{D}}$ it is enough to note that

$$
\sum_{k=0}^{\infty}\left(\sum_{n=1}^{\infty} \frac{1}{(n+k)(n+k+1)}\right)|\hat{f}(k)|=\sum_{k=0}^{\infty} \frac{|\hat{f}(k)|}{k+1} .
$$

Consequently, we also get the following

Corollary 2.2. The operator $H$ acts as a bounded operator from $H^{1}$ into $H^{p}$, $0<p<1$.

Theorem 2.3. If $f \in H^{1}$ is such that

$$
\int_{-\pi}^{\pi}\left|f\left(e^{i t}\right)\right| \log \frac{\pi}{|t|} d t<\infty
$$

then $H f \in H^{1}$.

Proof. We first show that if $f$ satisfies the assumptions, then the function $g(z)=$ $f(z) \log \frac{2}{1-z}$ is in $H^{1}$. To this end, we note that

$$
\int_{-\pi}^{\pi}\left|f\left(e^{i t}\right)\right| \log \frac{2}{\mid 1-e^{i t \mid}} d t=\int_{-\pi}^{\pi}\left|f\left(e^{i t}\right)\right| \log \frac{1}{\left|\sin \frac{t}{2}\right|} d t \leq \int_{-\pi}^{\pi}\left|f\left(e^{i t}\right)\right| \log \frac{\pi}{|t|} d t
$$

which implies that $g\left(e^{i t}\right)$ is in $L^{1}(\partial \mathbf{D})$. Since $g \in H^{p}, 0<p<1$, the Smirnov theorem (see, e.g., [9] p. 74) implies that $g$ is in $H^{1}$. Now using the formula (see [2]),

$$
H f(z)=\int_{0}^{1} \frac{f(r)}{1-r z} d r, \quad z \in \mathbf{D}
$$

and Fubini theorem we get

$$
\begin{aligned}
\frac{1}{2 \pi} \int_{0}^{2 \pi}\left|H f\left(e^{i t}\right)\right| d t & \leq \frac{1}{2 \pi} \int_{0}^{2 \pi} \int_{0}^{1} \frac{|f(r)| d r}{\mid 1-r e^{i t \mid}} d t=\int_{0}^{1}|f(r)| \frac{1}{2 \pi} \int_{0}^{2 \pi} \frac{d t}{\mid 1-r e^{i t \mid}} d r \\
& \leq C \int_{0}^{1}|f(r)| \log \frac{2}{1-r} d r
\end{aligned}
$$

Applying the Fejér-Riesz inequality to $g$, we see that $H f \in L^{1}(\partial \mathbf{D})$. Since $H f$ is in $H^{p}$ for $0<p<1$, the Smirnov theorem implies that $H f$ is in $H^{1}$.

2.1. The case of positive coefficients. If $\hat{f}(k) \geq 0$ for all $k$, then $H f$ is well defined by (1.2) or by (2.3) if and only if

$$
\sum_{k=0}^{\infty} \frac{\hat{f}(k)}{k+1}<\infty
$$

To see the "only if" part it is enough to take $z=0$. Furthermore, it is shown in [14] that if $\hat{f}(k) \downarrow 0$, then $f$ is in $H^{1}$ if and only if (2.4) holds. We use this fact to prove:

Theorem 2.4. If $\hat{f}(k) \geq 0$, then $H f \in H^{1}$ if and only if

$$
\sum_{n=0}^{\infty} \frac{\hat{f}(n) \log (n+2)}{n+1}<\infty
$$


Proof. The coefficients of $h=H f$ are given by

$$
\hat{h}(n)=\sum_{k=0}^{\infty} \frac{\hat{f}(k)}{n+k+1}
$$

and obviously $\hat{h}(n) \downarrow 0$ as $n \rightarrow \infty$. Hence, by what we mentioned above, $h \in H^{1}$ if and only if

$$
\sum_{n=0}^{\infty} \frac{1}{n+1} \sum_{k=0}^{\infty} \frac{\hat{f}(k)}{(n+k+1)}<\infty .
$$

Now note that this double sum is equal to

$$
\begin{aligned}
& \sum_{k=0}^{\infty} \hat{f}(k) \sum_{n=0}^{\infty} \frac{1}{(n+1)(n+k+1)} \\
& =\hat{f}(0) \sum_{n=0}^{\infty} \frac{1}{(n+1)^{2}}+\sum_{k=1}^{\infty} \frac{\hat{f}(k)}{k} \sum_{n=0}^{\infty}\left(\frac{1}{n+1}-\frac{1}{n+k+1}\right) \\
& =\hat{f}(0) \sum_{n=0}^{\infty} \frac{1}{(n+1)^{2}}+\sum_{k=1}^{\infty} \frac{\hat{f}(k)}{k} \sum_{n=0}^{k-1} \frac{1}{n+1}
\end{aligned}
$$

which implies the result.

Now let us consider the space $\mathfrak{B}^{1} \varsubsetneqq H^{1}$ defined by

$$
\mathfrak{B}^{1}=\left\{f \in H(\mathbf{D}): \int_{\mathbf{D}}\left|f^{\prime}(z)\right| d A(z)<\infty\right\} .
$$

It was also shown in [14] that if $\hat{f}(k) \downarrow 0$ then $f$ belongs to $\mathfrak{B}^{1}$ if and only if $(2.4)$ holds. This can be used to strengthen the statement that $H$ does not map $H^{1}$ into itself. More exactly we have

Proposition 2.5. The operator $H$ does not map $\mathfrak{B}^{1}$ into $H^{1}$.

Proof. By the above, the function

$$
f(z)=\sum_{n=2}^{\infty} \frac{z^{n}}{\log ^{3 / 2} n}
$$

belongs to $\mathfrak{B}^{1}$, while $H f$, by Theorem 2.4 , does not belong to $H^{1}$.

\section{Weighted Hardy spaces}

For $\alpha>0$ and $0<p \leq \infty$, we define the weighted Hardy spaces $H_{\alpha}^{p}$ as follows.

$$
H_{\alpha}^{p}=\left\{f \in H(\mathbf{D}): M_{p}(r, f)=O(1-r)^{-\alpha}\right\},
$$

The norm in these spaces is defined by

$$
\|f\|_{p, \alpha}=\sup _{0<r<1}(1-r)^{\alpha} M_{p}(r, f) .
$$

We start with the following

Theorem 3.1. If $\alpha+1 / p<1$, then the operator $H$ maps $H_{\alpha}^{p}$ into $H_{\alpha}^{p}$. 
Proof. Let $h=H f, f \in H_{\alpha}^{p}$. Then we have

$$
h^{\prime}(z)=\int_{0}^{1} \frac{r f(r) d r}{(1-r z)^{2}} .
$$

Using Minkowski's inequality, the inequality

$$
\int_{0}^{2 \pi}\left|1-\rho e^{i t}\right|^{-2 p} d t \asymp(1-\rho)^{1-2 p}
$$

and the inequality

$$
\left.|f(r)| \leq C(1-r)^{-\alpha-1 / p} \quad \text { (implied by } f \in H_{\alpha}^{p}\right)
$$

we get

$$
\begin{aligned}
M_{p}\left(\rho, h^{\prime}\right) & \leq C \int_{0}^{1}|f(r)|(1-\rho r)^{1 / p-2} d r \leq C \int_{0}^{1}(1-r)^{-\alpha-1 / p}(1-\rho r)^{1 / p-2} d r \\
& \leq C(1-\rho)^{-\alpha-1 / p} \int_{0}^{\rho}(1-r)^{1 / p-2} d r+C(1-\rho)^{1 / p-2} \int_{\rho}^{1}(1-r)^{-\alpha-1 / p} d r .
\end{aligned}
$$

Now the desired result is obtained by simple computation. It is enough to observe that $1 / p-2<-1$ and that $-\alpha-1 / p>-1$.

3.1. The case of monotone coefficients. Now our aim is to prove the following

Theorem 3.2. If $\{\widehat{f}(k)\}$ is a positive monotone sequence, then $f=\sum_{k=0}^{\infty} \widehat{f}(k) z^{k} \in$ $H_{\alpha}^{p}(1<p<\infty)$ if and only if

$$
\widehat{f}(k) \leq C(k+1)^{\alpha+1 / p-1} .
$$

Let

$$
\Delta_{n}(z)=\sum_{k \in I_{n}} z^{k}, \quad n \geq 0
$$

where

$$
I_{0}=\{0,1\}, \quad I_{n}=\left\{2^{n} \leq k \leq 2^{n+1}-1\right\}, \quad n \geq 1 .
$$

For $f \in H(\mathbf{D})$, let

$$
\Delta_{n} f(z)=\sum_{k \in I_{n}} \widehat{f}(k) z^{k}
$$

The following fact was proved in [11].

Lemma 3.3. Let $1<p<\infty$. A function $f \in H(\mathbf{D})$ is in $H_{\alpha}^{p}$ if and only if

$$
K(f):=\sup _{n} 2^{-n \alpha}\left\|\Delta_{n} f\right\|_{p}<\infty
$$

and we have $K(f) \asymp\|f\|_{p, \alpha}$.

Lemma 3.4. If $1<p<\infty$ and $\left\{\lambda_{n}\right\}$ is a positive monotone sequence, then

$$
\begin{aligned}
C^{-1} \lambda_{2^{n}}\left\|\Delta_{n}\right\|_{p} & \leq\left\|\sum_{k \in I_{n}} \lambda_{k} z^{k}\right\|_{p} \leq C \lambda_{2^{n+1}}\left\|\Delta_{n}\right\|_{p} \quad \text { if }\left\{\lambda_{n}\right\} \text { is increasing, } \\
C^{-1} \lambda_{2^{n+1}}\left\|\Delta_{n}\right\|_{p} & \leq\left\|\sum_{k \in I_{n}} \lambda_{k} z^{k}\right\|_{p} \leq C \lambda_{2^{n}}\left\|\Delta_{n}\right\|_{p} \quad \text { if }\left\{\lambda_{n}\right\} \text { is decreasing. }
\end{aligned}
$$


Proof. Since $\left\{z^{n}\right\}$ is a Schauder basis in $H^{p}, 1<p<\infty$, by Proposition 1.a.3 in [10], for any sequence $\left\{a_{k}\right\}$ and $0 \leq m \leq j<n$,

$$
\left\|\sum_{k=m}^{j} a_{k} z^{k}\right\|_{p} \leq C\left\|\sum_{k=m}^{n} a_{k} z^{k}\right\|_{p}
$$

where the constant $C$ is independent of $\left\{a_{k}\right\}, m, n$ and $j$.

By summation by parts,

$$
\sum_{k=m}^{n} \lambda_{k} a_{k} z^{k}=\sum_{k=m}^{n-1}\left(\lambda_{k}-\lambda_{k+1}\right) s_{k}+\lambda_{n} s_{n}
$$

where

$$
s_{k}=\sum_{j=m}^{k} a_{j} z^{j}
$$

Consequently,

$$
\left\|\sum_{k=m}^{n} \lambda_{k} a_{k} z^{k}\right\|_{p} \leq C\left(\sum_{k=m}^{n-1}\left|\lambda_{k}-\lambda_{k+1}\right|+\lambda_{n}\right)\left\|\sum_{k=m}^{n} a_{k} z^{k}\right\|_{p} .
$$

If $\left\{\lambda_{k}\right\}$ is monotonically decreasing, then the sum in brackets is $\lambda_{m}$, if $\left\{\lambda_{k}\right\}$ is monotonically increasing, this sum is $\left(\lambda_{n}-\lambda_{m}\right)+\lambda_{n} \leq 2 \lambda_{n}$. This proves the right-hand side inequalities. To prove the left inequalities we observe that if, for example, $\left\{\lambda_{k}\right\}$ increases, then $1 / \lambda_{k}$ decreases and by what we have already proved,

$$
\left\|\sum_{k=m}^{n} a_{k} z^{k}\right\|_{p}=\left\|\sum_{k=m}^{n} \frac{1}{\lambda_{k}}\left(\lambda_{k} a_{k} z^{k}\right)\right\|_{p} \leq C \frac{1}{\lambda_{m}}\left\|\sum_{k=m}^{n} \lambda_{k} a_{k} z^{k}\right\|_{p} .
$$

Proof of Theorem 3.2. Assume first that $1<p<\infty$. Since

$$
\left\|\Delta_{n}\right\|_{p}=\left\|1+z+\cdots+z^{2^{n}-1}\right\|_{p} \asymp 2^{n(1-1 / p)},
$$

Lemmas 3.3 and 3.4 imply that $f \in H_{\alpha}^{p}$ if and only if

$$
\widehat{f}\left(2^{n}\right) \leq C 2^{n(\alpha+1 / p-1)},
$$

and our claim follows from the monotonicity of $\{\widehat{f}(k)\}$.

3.2. Necessity of the condition $\alpha+1 / p<1$. To include in our considerations the cases $p=1, \infty$, we use polynomials $W_{n}$ (instead of $\Delta_{n}$ ) constructed in [7] (see also [13]). Let $\varphi$ be a $C^{\infty}$-function on $\mathbf{R}$ such that $\varphi(t)=1$ for $t \leq 1, \varphi(t)=0$ for $t \geq 2$, and $\varphi(t)$ is positive and decreasing on $(1,2)$. We set

$$
W_{0}(z)=1+z, \quad W_{n}(z)=\sum_{k \in J_{n}} \omega\left(k / 2^{n-1}\right) z^{k}, \quad n \geq 1
$$

where

$$
J_{n}=\left\{k \in \mathbf{N}: 2^{n-1} \leq k \leq 2^{n+1}\right\}
$$

and

$$
\omega(t)=\varphi(t / 2)-\varphi(t)
$$


The convolution $f * g$ of two functions $f, g \in H(\mathbf{D})$ is defined by

$$
f * g(z)=\sum_{n=0}^{\infty} \widehat{f}(n) \widehat{g}(n) z^{n}
$$

where $f(z)=\sum_{n=0}^{\infty} \widehat{f}(n) z^{n}$ and $g(z)=\sum_{n=0}^{\infty} \widehat{g}(n) z^{n}$.

The following inequality was proved in [6] and [7].

$$
\left\|W_{n} * f\right\|_{p} \leq C\|f\|_{p}, \quad n=0,1,2, \ldots, \quad 0<p \leq \infty .
$$

We will also need the following lemmas.

Lemma 3.5. [8] Let $0<p \leq \infty$. A function $f \in H(\mathbf{D})$ is in $H_{\alpha}^{p}$ if and only if $\left\|W_{n} * f\right\|_{p}=O\left(2^{n \alpha}\right)$, and we have

$$
\|f\|_{p, \alpha} \asymp \sup _{n} 2^{-n \alpha}\left\|W_{n} * f\right\|_{p} .
$$

Lemma 3.6. [13, Exercise 7.3.5] Let $p \in(0, \infty], P(z)=\sum_{k=m}^{4 m} a_{k} z^{k}, Q(z)=$ $\sum_{k=m}^{4 m}(k+1)^{\beta} a_{k} z^{k}$, where $m$ is a positive integer and $\beta \in \mathbf{R}$. Then there is a constant $C=C(p, \beta)$ such that

$$
C^{-1} m^{\beta}\|P\|_{p} \leq\|Q\|_{p} \leq C m^{\beta}\|P\|_{p}
$$
to $\beta$.

Moreover, for $|\beta|<\frac{1}{2}$ the constants $C(p, \beta)$ are uniformly bounded with respect

Proof. Let $W_{m}$ be a trigonometric polynomial such as in Lemma 7.3.2 in [13] with $\psi(x)=(x+1 / m)^{\beta} \varphi(x)$, where $\varphi$ is a $C^{\infty}$-function such that $\operatorname{supp}(\varphi) \subset\left(\frac{1}{2}, 5\right)$ and $\varphi(x)=1$ for $x \in[1,4]$. Then

$$
W_{m} * P(z)=m^{-\beta} Q(z) .
$$

Our claim will follow from Theorem 7.3.4 in [13] if we can find the constant $C_{N}$ in Lemma 7.3.2 [13] independent of $\beta$ and $m$. But, since $\operatorname{supp}(\varphi) \subset\left(\frac{1}{2}, 5\right)$, the Leibniz formula

$$
\psi^{(N)}(x)=\sum_{j=0}^{N}\left(\begin{array}{c}
N \\
j
\end{array}\right) \beta(\beta-1) \ldots(\beta-j+1)\left(x+\frac{1}{m}\right)^{\beta-j} \varphi^{(N-j)}(x)
$$

implies that $\left|\psi^{(N)}(x)\right|$ is bounded uniformly with respect to $\beta$ and $m$ and the claim follows.

To prove the last statement it is enough to show that

Lemma 3.7. For $p \in[1, \infty]$ we have

$$
\left\|W_{n}\right\|_{p} \asymp 2^{n(1-1 / p)} .
$$

Proof. The case $p=\infty$ is easy. Assume that $1 \leq p<\infty$. Since

$$
M_{\infty}\left(r, W_{n}\right) \leq C(1-r)^{-1 / p}\left\|W_{n}\right\|_{p}
$$

and $M_{\infty}\left(r, W_{n}\right) \geq r^{2^{n+1}}\left\|W_{n}\right\|_{\infty}$, taking $r=1-2^{-n-1}$, we obtain

$$
C\left\|W_{n}\right\|_{p} \geq 2^{n(1-1 / p)} .
$$


To prove the reverse inequality, we take $f(z)=(1-z)^{-2}$ and use Lemma 3.1 in [11] and (3.2) to obtain

$$
r^{2^{n+1}}\left\|\sum_{k \in J_{n}}(k+1) \widehat{W}_{n}(k) z^{k}\right\|_{p} \leq M_{p}\left(r, W_{n} * f\right)=\left\|W_{n} * f_{r}\right\|_{p} \leq C(1-r)^{-2+1 / p} .
$$

Taking $r=1-2^{-n-1}$ we get

$$
\left\|\sum_{k \in J_{n}}(k+1) \widehat{W}_{n}(k) z^{k}\right\|_{p} \leq C 2^{(2-1 / p) n}
$$

and the result follows from Lemma 3.6

We will show that if the condition $\alpha+1 / p<1$ is not satisfied, then the operator $H$ cannot be extended as a continuous operator even into the space $H(\mathbf{D})$. More exactly, we have

Theorem 3.8. If $\alpha+1 / p \geq 1, \alpha>0$ and $p \geq 1$, then the operator $H$ cannot be extended to a continuous operator from $H_{\alpha}^{p}$ into $H(\mathbf{D})$.

Proof. For $\beta \in\left(0, \frac{1}{2}\right)$ set

$$
f_{\beta}(z)=\sum_{k=0}^{\infty}(k+1)^{-\beta} z^{k}
$$

Then Lemmas 3.6 and 3.7, and the assumption $\alpha \geq 1-1 / p$ imply that

$$
\left\|W_{n} * f_{\beta}\right\|_{p} \leq C 2^{-n \beta}\left\|W_{n}\right\|_{p} \leq C 2^{n(-\beta+1-1 / p)} \leq C 2^{n(-\beta+\alpha)} \leq C 2^{n \alpha} .
$$

By Lemma 3.5 this means that

$$
\sup _{0<\beta<\frac{1}{2}}\left\|f_{\beta}\right\|_{p, \alpha}<\infty
$$

If $H$ could be extended to a bounded operator from $H_{\alpha}^{p}$ into $H(\mathbf{D})$, then we would have $\sup _{0<\beta<\frac{1}{2}}\left|H f_{\beta}(0)\right|<\infty$, because $f \mapsto f(0)$ is a continuous linear operator on $H(\mathbf{D})$. However,

$$
H f_{\beta}(0)=\sum_{k=0}^{\infty} \frac{1}{(k+1)^{\beta+1}}
$$

and $\lim _{\beta \rightarrow 0} H f_{\beta}(0)=\infty$. This contradiction proves the result.

\section{Logarithmically weighted Bergman spaces}

It is known that the Hilbert matrix does not act on $A^{2}$ (see [3]). For $\alpha>0$ we define the logarithmically weighted Bergman space $A_{\log _{\alpha}}^{2} \subset A^{2}$ as follows.

$$
A_{\log ^{\alpha}}^{2}=\left\{f \in H(\mathbf{D}):\|f\|_{\log ^{\alpha}}^{2}=\int_{\mathbf{D}}|f(z)|^{2}\left(\log \frac{2}{1-|z|^{2}}\right)^{\alpha} d A(z)<\infty\right\},
$$

where $d A(z)$ is the area measure on $\mathbf{D}$ normalized so that $\int_{\mathbf{D}} d A(z)=1$. The following lemma can be proved in a standard way. 
Lemma 4.1. If $f \in A_{\log ^{\alpha}}^{2}, \alpha>0$, then there exists a constant $C>0$ such that

$$
|f(z)| \leq \frac{C\|f\|_{\log ^{\alpha}}}{\left(1-|z|^{2}\right)\left(\log \frac{2}{1-|z|^{2}}\right)^{\frac{\alpha}{2}}}
$$

for every $z \in \mathbf{D}$.

We claim that $H$ is well defined on $A_{\log ^{\alpha}}^{2}$ for $\alpha>2$. This follows from the following

Lemma 4.2. Let $\alpha>2$. If $f \in A_{\log ^{\alpha}}^{2}$, then

$$
\sum_{k=0}^{\infty} \frac{|\hat{f}(k)|}{k+1} \leq C\|f\|_{\log ^{\alpha}} .
$$

Proof. Since the function $s \mapsto M_{2}(s, f)$ is increasing on $[0,1)$, the Chebyshev inequality implies

$$
\begin{aligned}
\|f\|_{\log ^{\alpha}}^{2} & =\int_{0}^{1} M_{2}^{2}(s, f)\left(\log \frac{2}{1-s^{2}}\right)^{\alpha} s d s \geq \frac{1}{2} \int_{r}^{1} M_{2}^{2}(s, f)\left(\log \frac{2}{1-s^{2}}\right)^{\alpha} d s \\
& \geq \frac{1}{2}(1-r)\left(\log \frac{2}{1-r^{2}}\right)^{\alpha} M_{2}^{2}(r, f) .
\end{aligned}
$$

This means that for $r \in[0,1)$,

$$
\sum_{k=0}^{\infty}|\hat{f}(k)|^{2} r^{2 k} \leq C\|f\|_{\log ^{\alpha}}^{2}(1-r)^{-1}\left(\log \frac{2}{1-r}\right)^{-\alpha} .
$$

Taking $r=1-1 / m$, we get

$$
\sum_{k=m}^{2 m}|\hat{f}(k)|^{2} \leq C\|f\|_{\log ^{\alpha}}^{2} m(\log 2 m)^{-\alpha}
$$

Consequently, for $\alpha>2$, we have

$$
\begin{aligned}
\sum_{k=1}^{\infty} \frac{|\hat{f}(k)|}{k+1} & =\sum_{k=1}^{\infty} \sum_{j=2^{k-1}}^{2^{k}-1} \frac{|\hat{f}(j)|}{j+1} \leq \sum_{k=1}^{\infty} 2^{1-k} \sum_{j=2^{k-1}}^{2^{k}-1}|\hat{f}(j)| \\
& \leq \sum_{k=1}^{\infty} 2^{1-k} 2^{\frac{k-1}{2}}\left(C\|f\|_{\log ^{\alpha}}^{2} 2^{k-1}\left(\log 2^{k}\right)^{-\alpha}\right)^{\frac{1}{2}} \\
& =C\|f\|_{\log ^{\alpha}} \sum_{k=1}^{\infty} \frac{1}{k^{\frac{\alpha}{2}}} \leq C\|f\|_{\log ^{\alpha}} .
\end{aligned}
$$

Moreover, for $\alpha>2$ the Hilbert matrix operator acting on $A_{\log ^{\alpha}}^{2}$ can also be expressed in the integral form (2.3). Furthermore, we have

Theorem 4.3. If $\alpha>3$, then $H$ acts as a bounded operator from $A_{\log ^{\alpha}}^{2}$ to $A^{2}$. 
Proof. From (2.3) and the integral form of Minkowski's inequality we obtain

$$
\begin{aligned}
\|H f\|_{A^{2}} & =\left(\int_{\mathbf{D}}|H f(z)|^{2} d A(z)\right)^{\frac{1}{2}} \leq\left(\int_{\mathbf{D}}\left(\int_{0}^{1} \frac{|f(r)|}{|1-r z|} d r\right)^{2} d A(z)\right)^{\frac{1}{2}} \\
& \leq \int_{0}^{1}|f(r)|\left(\int_{\mathbf{D}} \frac{d A(z)}{|1-r z|^{2}}\right)^{\frac{1}{2}} d r \leq C \int_{0}^{1}|f(r)|\left(\log \frac{2}{1-r^{2}}\right)^{\frac{1}{2}} d r .
\end{aligned}
$$

By Lemma 4.1,

$$
\|H f\|_{A^{2}} \leq C \int_{0}^{1} \frac{d r}{\left(1-r^{2}\right)\left(\log \frac{2}{1-r^{2}}\right)^{\frac{\alpha-1}{2}}}\|f\|_{\log ^{\alpha}},
$$

and the last integral converges for $\alpha>3$.

\section{The Bloch and Besov spaces}

For $1<p \leq \infty$, let $\mathcal{B}_{p}$ denote the analytic Besov space consisting of functions $f \in H(\mathbf{D})$ for which

$$
\|f\|_{\mathcal{B}_{p}}:=|f(0)|+\left(\int_{\mathbf{D}}\left|f^{\prime}(z)\right|^{p}\left(1-|z|^{2}\right)^{p-2} d A(z)\right)^{1 / p}<\infty .
$$

In the case $p=\infty$ this is understood as

$$
\|f\|_{\mathcal{B}_{\infty}}=|f(0)|+\sup _{z \in \mathbf{D}}\left(1-|z|^{2}\right)\left|f^{\prime}(z)\right|<\infty,
$$

and hence $\mathcal{B}_{\infty}=\mathcal{B}$ is the Bloch space. The reader is referred to, e.g., [15] for results on these spaces.

It is easy to check that if $f(z)=\log \frac{1}{1-z}$, then

$$
H f(z)=\sum_{n=0}^{\infty} \frac{1}{n+1}\left(1+\frac{1}{2}+\cdots+\frac{1}{n+1}\right) z^{n} .
$$

This shows that Bloch space $\mathcal{B}$ is not mapped into itself.

The following lemma describes a space of analytic functions in $\mathbf{D}$ that are mapped by $H$ into the Bloch space.

Proposition 5.1. If $f \in H(\mathbf{D})$ satisfies the condition

$$
\sup _{z \in \mathbf{D}}\left|f^{\prime}(z)\right|(1-|z|)\left(\log \frac{2}{1-|z|}\right)^{1+\varepsilon}<\infty
$$

for an $\varepsilon>0$, then $H f \in \mathcal{B}$.

Proof. Assume that $f \in H(\mathbf{D})$ satisfies (5.1) and set

$$
F(z)=f(z)-f(0) .
$$

It is enough to show that $H F \in \mathcal{B}$. Clearly, $F$ also satisfies (5.1). Then by Lemma 4.2 .8 in [15] we can write

$$
F(z)=\int_{\mathbf{D}} \frac{F^{\prime}(w)\left(1-|w|^{2}\right)}{\bar{w}(1-\bar{w} z)^{2}} d A(w)
$$


Consequently,

$$
\begin{aligned}
\left|(H F)^{\prime}(z)\right| & \leq C \int_{0}^{1} \int_{\mathbf{D}} \log ^{-1-\varepsilon}\left(\frac{2}{1-|w|}\right) \frac{1}{|1-\bar{w} r|^{2}|1-r z|^{2}} d A(w) d r \\
& \leq C \int_{0}^{1} \int_{0}^{1} \log ^{-1-\varepsilon}\left(\frac{2}{1-s}\right) \frac{d s d r}{(1-s r)(1-r|z|)^{2}} \\
& \leq \frac{C}{1-|z|} \int_{0}^{1} \log ^{-1-\varepsilon}\left(\frac{2}{1-s}\right) \frac{1}{1-s} d s
\end{aligned}
$$

Since the last integral is finite, our claim is proved.

On the other hand, we have

Proposition 5.2. If $f \in \mathcal{B}$, then

$$
\left|(H f)^{\prime}(z)\right| \leq C \frac{1}{1-|z|} \log \frac{2}{1-|z|} .
$$

Proof. For $f \in \mathcal{B}$ set

$$
A_{n}(f)=\sum_{k=0}^{\infty} \frac{\widehat{f}(k)}{n+k+1}=\int_{\mathbf{D}} \frac{f(z)}{1-\bar{z}}|z|^{2 n} d A(z) .
$$

Assuming additionally that $f(0)=0$ and using the Fubini theorem, we obtain

$$
\begin{aligned}
\left|A_{n}(f)\right| & \leq\left|\int_{\mathbf{D}} \int_{\mathbf{D}} \frac{f^{\prime}(w)\left(1-|w|^{2}\right)}{\bar{w}(1-\bar{w} z)^{2}} d A(w) \frac{|z|^{2 n}}{1-\bar{z}} d A(z)\right| \\
& =\left|\int_{\mathbf{D}} \frac{f^{\prime}(w)\left(1-|w|^{2}\right)}{\bar{w}} \int_{0}^{1}\left(\frac{1}{\pi} \int_{0}^{2 \pi} \frac{d \theta}{\left(1-\bar{w} r e^{i \theta}\right)^{2}\left(1-r e^{-i \theta}\right)}\right) r^{2 n+1} d r d A(w)\right| \\
& \leq C \int_{0}^{1} r^{2 n+1} \int_{\mathbf{D}} \frac{d A(w)}{\left|1-r^{2} \bar{w}\right|^{2}} d r \leq C \int_{0}^{1} \log \frac{2}{\left(1-r^{2}\right)} r^{2 n+1} d r \\
& \leq C \int_{0}^{1} r^{n}\left(\log 2+\sum_{k=1}^{\infty} \frac{r^{k}}{k}\right) d r \leq C \frac{1}{n+1}\left(1+\frac{1}{2}+\cdots+\frac{1}{n+1}\right)
\end{aligned}
$$

Hence

$$
\begin{aligned}
\left|(H f)^{\prime}(z)\right| & =\left|\sum_{n=1}^{\infty} n A_{n}(f) z^{n-1}\right| \leq \sum_{n=1}^{\infty} n\left|A_{n}(f)\right||z|^{n-1} \\
& \leq C \sum_{n=0}^{\infty}\left(1+\frac{1}{2}+\ldots \frac{1}{n+1}\right)|z|^{n} \leq C \frac{1}{1-|z|} \log \frac{2}{1-|z|}
\end{aligned}
$$

The example of $f(z)=\log \frac{1}{1-z}$ shows that the inequality in the last lemma cannot be improved.

A little bit more complicated calculations give the following

Proposition 5.3. If $f \in \mathcal{B}_{p}, 1<p<\infty$, then

$$
\left|(H f)^{\prime}(z)\right| \leq C \frac{1}{1-|z|}\left(\log \frac{2}{1-|z|}\right)^{\frac{1}{p^{\prime}}},
$$

where $\frac{1}{p}+\frac{1}{p^{\prime}}=1$. 
Proof. Using the notation from the proof of Proposition 5.2 under the assumption that $f(0)=0$ we get, in much the same way as above,

$$
\begin{aligned}
\left|A_{n}(f)\right| & \leq C \int_{0}^{1} r^{2 n+1} \int_{\mathbf{D}} \frac{\left|f^{\prime}(w)\right|\left(1-|w|^{2}\right)}{\left|1-r^{2} \bar{w}\right|^{2}} d A(w) d r \\
& \leq C\|f\|_{\mathcal{B}_{p}} \int_{0}^{1} r^{2 n+1}\left(\log \frac{2}{1-r^{2}}\right)^{\frac{1}{p^{\prime}}} d r .
\end{aligned}
$$

Hence

$$
\begin{aligned}
\left|A_{n}(f)\right|^{p^{\prime}} & \leq C\|f\|_{\mathcal{B}_{p}}^{p^{\prime}}\left(\int_{0}^{1} r^{2 n+1} d r\right)^{p^{\prime}-1} \int_{0}^{1} r^{2 n+1} \log \frac{2}{1-r^{2}} d r \\
& \leq C\|f\|_{\mathcal{B}_{p}}^{p^{\prime}}\left(\frac{1}{n+1}\right)^{p^{\prime}}\left(1+\frac{1}{2}+\cdots+\frac{1}{n+1}\right) .
\end{aligned}
$$

Consequently,

$$
\begin{aligned}
\left|(H f)^{\prime}(z)\right|^{p^{\prime}} & \leq\left(\sum_{n=0}^{\infty}(n+1)\left|A_{n+1}(f) \| z\right|^{n}\right)^{p^{\prime}} \\
& \leq\left(\sum_{n=0}^{\infty}(n+1)^{p^{\prime}}\left|A_{n+1}(f)\right|^{p^{\prime}}|z|^{n}\right)\left(\sum_{n=0}^{\infty}|z|^{n}\right)^{p^{\prime}-1} \\
& \leq C\|f\|_{\mathcal{B}_{p}}^{p^{\prime}}\left(\frac{1}{1-|z|}\right)^{p^{\prime}-1}\left(\sum_{n=0}^{\infty}\left(1+\frac{1}{2}+\cdots+\frac{1}{n+1}\right)|z|^{n}\right) \\
& \leq C\|f\|_{\mathcal{B}_{p}}^{p^{\prime}}\left(\frac{1}{1-|z|}\right)^{p^{\prime}} \log \frac{2}{1-|z|},
\end{aligned}
$$

which proves our claim.

\section{References}

[1] Diamantopoulos, E.: Hilbert matrix on Bergman spaces. - Illinois J. Math. 48:3, 2004, 1067-1078.

[2] Diamantopoulos, E., and A. G. Siskakis: Composition operators and the Hilbert matrix. - Studia Math. 140:2, 2000, 191-198.

[3] Dostanić, M., M. Jevtić, and D. Vukotić: Norm of the Hilbert matrix on Bergman and Hardy spaces and a theorem of Nehari type. - J. Funct. Anal. 254, 2008, 2800-2815.

[4] Duren, P. L.: Theory of $H^{p}$ spaces. - Pure Appl. Math. 38, Academic Press, New York, 1970.

[5] Hardy, G. H., J. E. Littlewood, and G. Pólya: Inequalities. - Cambridge Univ. Press, Cambridge, 1934.

[6] Jevtić, M., and M. Pavlović: On Hahn-Banach extension property in Hardy and mixed norm spaces on the unit ball. - Monatsh. Math. 11, 1991, 137-145.

[7] Jevtić, M., and M. Pavlović: On multipliers from $H^{p}$ into $l^{q}, 0<p<q<1$. - Arch. Math. $56,1991,174-180$.

[8] Jevtić, M., and M. Pavlović: Coefficient multilpliers on spaces of analytic functions. - Acta Sci. Math. (Szeged) 64, 1998, 531-545.

[9] Koosis, P.: Introduction to $H^{p}$ spaces. - Cambridge Univ. Press, Cambridge, 1998. 
[10] Linderstrauss, J., and L. Tzafriri: Classical Banach spaces I. Sequence spaces. - SpringerVerlag, Berlin-New York, 1977.

[11] Mateljević, M., and M. PAvlović: $L^{p}$ behaviour of the integral means of analytic functions. - Studia Math. 77, 1984, 219-237.

[12] Nowak, M., and M. Pavlović: On the Libera operator. - J. Math. Anal. Appl. 370, 2010, $588-599$.

[13] Pavlović, M.: Introduction to function spaces on the disk. - Matematički institut SANU, Beograd, 2004.

[14] Pavlović, M.: Analytic functions with decreasing coefficients and Hardy spaces. - To appear.

[15] ZHU, K.: Operator theory in function spaces. - Dekker, New York, 1990.

Received 18 March 2011 